
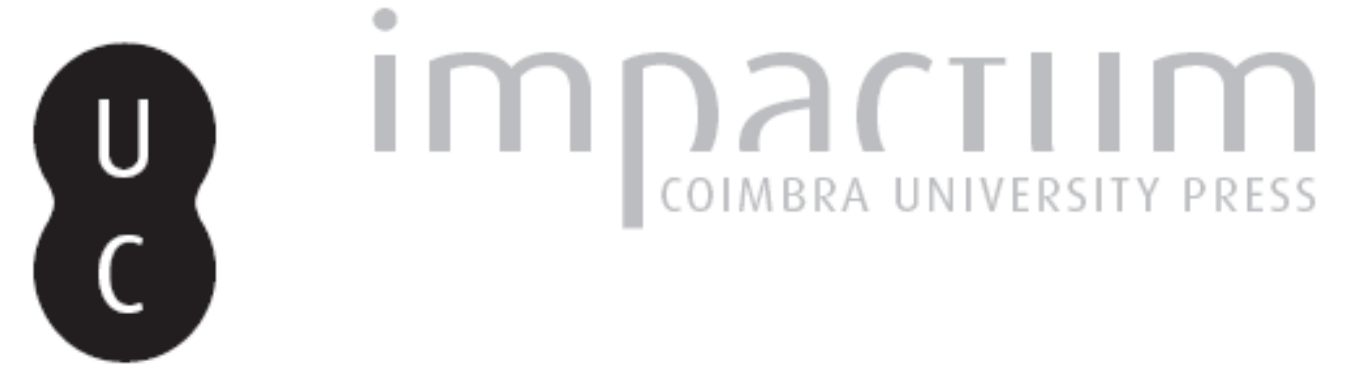

\title{
Le risque en montagne: les avalanches dans les montagnes françaises
}

\section{Autor(es): $\quad$ Veyret, Yvette}

Publicado por: Associação Portuguesa de Riscos, Prevenção e Segurança

URL persistente:

URI:http://hdl.handle.net/10316.2/40096

DOI:

DOI:https://doi.org/10.14195/1647-7723_8_1

Accessed : $\quad$ 26-Apr-2023 04:44:47

A navegação consulta e descarregamento dos títulos inseridos nas Bibliotecas Digitais UC Digitalis, UC Pombalina e UC Impactum, pressupõem a aceitação plena e sem reservas dos Termos e Condições de Uso destas Bibliotecas Digitais, disponíveis em https://digitalis.uc.pt/pt-pt/termos.

Conforme exposto nos referidos Termos e Condições de Uso, o descarregamento de títulos de acesso restrito requer uma licença válida de autorização devendo o utilizador aceder ao(s) documento(s) a partir de um endereço de IP da instituição detentora da supramencionada licença.

Ao utilizador é apenas permitido o descarregamento para uso pessoal, pelo que o emprego do(s) título(s) descarregado(s) para outro fim, designadamente comercial, carece de autorização do respetivo autor ou editor da obra.

Na medida em que todas as obras da UC Digitalis se encontram protegidas pelo Código do Direito de Autor e Direitos Conexos e demais legislação aplicável, toda a cópia, parcial ou total, deste documento, nos casos em que é legalmente admitida, deverá conter ou fazer-se acompanhar por este aviso.

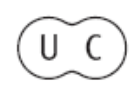




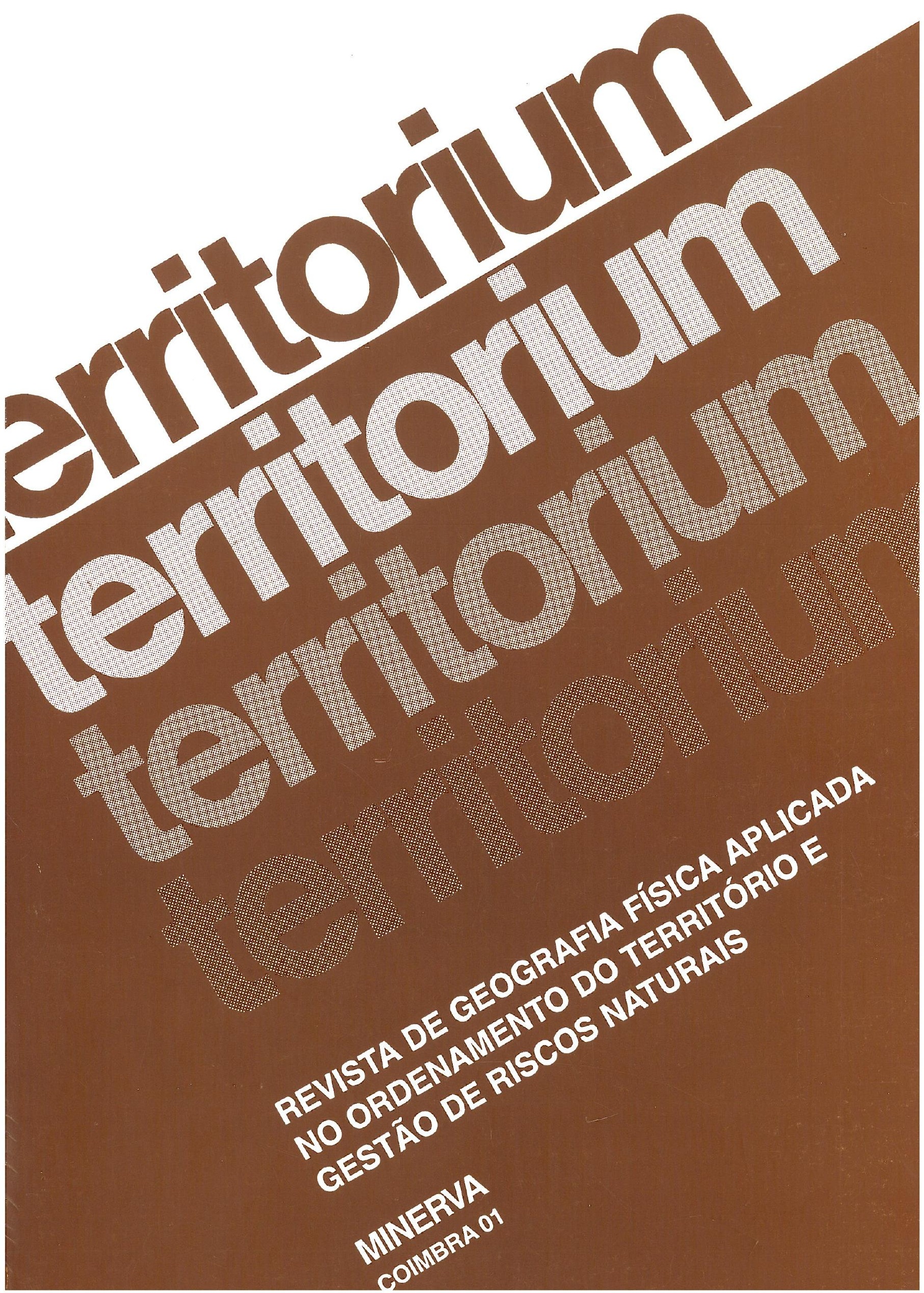




\title{
Le risque en montagne: les avalanches dans les montagnes françaises
}

\author{
Yvette Veyret *
}

\begin{abstract}
Resumo:
Apresentam-se em primeiro lugar alguns exemplos de avalanches ocorridas em montanhas francesas. Destacam-se, seguidamente, as situações que favorecem as avalanches, o modo como se faz a sua previsão, os diversos trabalhos de protecção e, finalmente, os estudos que permitem a prevenção.

Palavras chave:

Riscos naturais, riscos em montanha, avalanches.

Résumé:

Quelques exemples d'avalanches sont présentes. Ensuite, on souligne les situations favorables aux avalanches, la façon dont est faite la prévision, les travaux de protection et, finalemment, les études permettant la prévention. Mots clés:

Risques naturels, risques en montagne, avalanches.
\end{abstract}

\section{Abstract:}

First of all, the Author presents some examples of avalanches in the France mountains. Then, emphasizes the situations influencing avalanches, as well as its forecasting, protection and prevention.

Key words:

Natural hazard and risk, mountain hazards, avalanches.

\section{Introduction}

Le risque naturel est devenu une préoccupation majeure des pouvoirs publics français depuis la création en 1984 de la Délégation aux risques majeurs. Le risque désigne un danger, et recouvre à la fois l'aléa, c'est à dire le processus physique et la vulnérabilité des biens et des personnes.

Les aléas et par tant les risques se définissent en terme de fréquence et d'intensité, ce qui impose de réaliser un travail historique approfondi pour retrouver la chronologie des processus du même type survenus dans un secteur donné, et pour établir l'ampleur des phénomènes, autrement dit leur intensité. De tels

\footnotetext{
* Professeur de Géographie à I'Université de Paris X. j.veyret@wanadoo.fr
}

travaux conduisent à envisager en terme statistique un temps de retour possible des aléas.

Le risque d'avalanchen'est qu'un aspect du risque en montagne. La pente, l'importance des précipitations, l'instabilité de certaines couvertures de formations superficielles, la torrentialité, expliquent que la montagne soit un milieu fortement affecté par des morphodynamiques diverses. Or, ces processus peuvent concerner les populations qui fréquentent l'espace montagnard.

L'étude des avalanches en montagne, processus naturels liés à la fois aux caractères de l'enneigement (épaisseur, hétérogénéité du manteau nival) et à la pente. peut s'effectuer sous plusieurs angles et selon diverses finalités. Les géographes ont longtemps envisagé les avalanches en terme morphodynamique, pour analyser le rôle de la neige. Mais les avalanches s'inscrivent aussi dans une réflexion sur l'aménagement 
des territoires montagnards, dans la mesure où elles présentent des dangers pour les populations qui vivent et fréquentent ces territoires. Dans le domaine alpin européen, vivent 11 millions de personnes, mais au cours des périodes touristiques d'hiver et d'été, ce nombre peut être multiplié par deux ou trois.

En France, les avalanches ne sont pas à l'origine du plus grand nombre de dégâts et de victimes, elles se placent très loin derrière les inondations. Cependant, quand survient une avalanche, la perception fortement négative du public affecte l'image de la station concernée et l'on sait le poids de cette image en terme de fréquentation. Le risque d'avalanches à la hors-piste, les autres sont affectées sur des voies de circulation, dans des habitations.

Le bilan des vingt dernières années indique que la hausse spectaculaire de la fréquentation en montagne n'a pas provoqué une augmentation sensible du nombre d'accidents.

Les accidents de la saison 98-99 montrent que géographiquement parlant, les Alpes du nord sont les plus touchées parce qu'elles regroupent le plus grand nombre de stations et le plus grand nombre d'utilisateurs.

Tableau $\mathrm{n}^{\circ} 1$

Les avalanches récentes dans les Alpes françaises. Les hivers particulièrement avalancheux.

1970 à Val d'Isère dans les Alpes, une avalanche de neige poudreuse se produisit, résultant d'une forte chute ( 3 à $4 \mathrm{~m}$ entre le 4 et le 10 février), sur une sous couche sans cohésion. Il s'est agi d'une avalanche à aérosol qui a pénétré dans un bâtiment et provoqué 39 victimes. De nombreuses coulées de neige se sont manifestées ailleurs dans le massif alpin, des maisons furent touchées à Tignes, Chamonix, Modane.

1978 l'enneigement a été faible jusqu'au 10 janvier, $2 \mathrm{~m}$ de neige tombent ensuite sur les Alpes du Sud. Les toits s'effondrent sous la charge de neige, des avalanches se déclenchent. Puis les Alpes du Nord sont touchées: il y a plusieurs morts, cinq chalets sont endommagés dans la vallée de Chamonix, les dégâts à la forêt sont nombreux.

1981 avalanches en Savoie, en Isère: dix morts, et des dégâts à la forêt. Plus de cinquante maisons sont détruites.

1999 dans la vallée de Chamonix nombreuses avalanches de grande ampleur, des trajectoires inhabituelles ont été empruntées (des chalets sont atteints en zone blanche du Plan de Prévention des Risques) D'autres secteurs des Alpes ont également été affectés.

différence des autres types de risques (inondations) est largement un risque individuel lié aux loisirs. La hausse considérable du tourisme en montagne demande de développer des mesures de prévention et de prévision. Sur trente ans la montagne a provoqué en moyenne une trentaine de morts par an, dont 11 par avalanches. Il est nécessaire de rapprocher ces chiffres du nombre d'accidents de la route dans le même temps!

\section{Les victimes des avalanches en France}

Un suivi précis des accidents liés aux avalanches est effectué depuis 1971 par l'Association Nationale pourl'Etude de la Neige et des Avalanches (ANENA), il ne s'agit que des accidents graves, les chiffres ne prennent pasen compte les avalanches qui se produisent sans faire de victimes. D'octobre 1971 à 1994, pour 857 accidents graves, le nombre de personnes emportées s'élève à 2600 , et le nombre de victimes décédées à 670 .

Les personnes concernées sont celles qui s'adonnent à des activités récréatives, et principalement au ski
Tableau n" 2

Accidents et localisation des avalanches hiver 1998-1999

\begin{tabular}{|lcc|}
\hline Activités & $\begin{array}{c}\text { nombre } \\
\text { d'accidents }\end{array}$ & $\begin{array}{c}\text { décès } \\
\text { et blessés }\end{array}$ \\
\hline Hors piste & 24 & 20 \\
Snowbord hors piste & 13 & 18 \\
Piste & 3 & 2 \\
Randonnée ski & 11 & 21 \\
Autres & 9 & 23 \\
\hline Total & 60 & 84 \\
\hline
\end{tabular}

Pour les 60 accidents, la répartition géographique est la suivante:

\begin{tabular}{|lcc|}
\hline Alpes du Nord & & \\
Haute Savoie & 10 & 38 \\
Savoie & 21 & 23 \\
Isère & 8 & 7 \\
Alpes du Sud & & \\
Haute-Alpes & 16 & 13 \\
Alpes de haute Provence & 3 & 1 \\
Jura & 1 & 2 \\
Pyrénées atlantiques & 1 & 0 \\
\hline
\end{tabular}




\section{Lessituations avalancheuses, l'état du manteau nival}

Les avalanches sont un phénomène spécifiquement montagnard, lié à la fois à la gravité et à la présence de neige. Au-delà d'une altitude qui varie selon les massifs et l'exposition des pentes, le coefficient de nivosité s'établit à $100 \%$, c'est le cas au-dessus de $3500 \mathrm{~m}$ d'altitude dans le massif du Mont Blanc, de $3800 \mathrm{~m}$ en Vanoise en raison de la position plus interne du massif. Le coefficient nivométrique atteint $60 \%$ à $2000 \mathrm{~m}, 75 \%$ à $2500 \mathrm{~m}, 90 \%$ à $3000 \mathrm{~m}$ et $100 \%$ au-dessus.

On distingue les couches de neige sèche, légère (masse volumique entre 20 et $100 \mathrm{~kg} / \mathrm{m}^{3}$ ), peu cohérente et les couches humides collantes et lourdes (masse volumique voisine de $150 \mathrm{~kg} / \mathrm{m}^{3}$ jusqu'à $\left.300 \mathrm{~kg} / \mathrm{m}^{3}\right)$.

Le vent modifie la nature de la neige, il brise les cristaux en les roulant et les transforme en grains fins. La neige se tasse et devient plus lourde (200 à $300 \mathrm{~kg} / \mathrm{m}^{3}$ )

\section{On distingue:}

- des avalanches de neige dense, lourde qui peuvent mobiliser toute l'épaisseur du manteau nival jusqu'au substrat. A l' écoulement laminaire du manteau au début de sa mise en mouvement, succèdent des boules glissant les unes contre les autres, globalement le déplacement est lent et la neige avance à la manière d'un bulldozer avec une pression de plusieurs tonnes au $\mathrm{m}^{3}$. Ces avalanches affectent fréquemment des couloirs qu'elles contribuent à modeler, elles surviennent le plus souvent au printemps, lors des redoux.

- des avalanches de neige poudreuse qui incorpore de l'air pour donner un aérosol, ont une faible densité qui leur permet de dévaler les pentes et de se déplacer à contre pente sur le versant d'en face à une vitesse élevée $(50$ à $100 \mathrm{~m} / \mathrm{s})$. Elles sont précédées par un souffle souvent très destructeur et se produisent après de fortes chutes de neige peu cohérente survenues par temps froid. C'est la surcharge due à la dernière neige tombée qui provoque le déclenchement.

- les ruptures de plaques à vent, peuvent déclencher des avalanches.

Le rôle du vent est déterminant dans la redistribution de la neige, il forme des congères et des plaques à vent. Celles-ci sont en principe connues et répertoriées car elles apparaissent en fonction de la topographie et de la direction du vent, dans des sites aisés à repérer et à cartographier.

L'instabilité du manteau nival résulte plus encore que de son épaisseur, de sa fréquente hétérogénéité, due à la superposition de couches de caractères différents.

\section{L’hétérogénéité du manteau nival}

Le manteau nival peut traduire la mémoire des chutes. Les différentes strates constituent à un moment donné, un bilan de l'histoire météorologique des jours et des semaines qui précèdent.

Une coupe effectuée dans le couvert nival révèle souvent un aspect feuilleté. Chaque couche correspond à une chute de neige dont les cristaux ont subi une transformation nommée "métamorphose" fonction des conditions locales.

Il existe des métamorphoses d'isothermie qui se manifestent par tassement dans une couche à température homogène proche de $0^{\circ}$. Les cristaux sous l'effet de leur propre poids se tassent, se transforment en grains fins arrondis et soudés entre eux. La neige est alors plus dense, la couche initiale a perdu les deux tiers de son épaisseur.

Quand existe une forte différence de température entre la base et la surface, se développe une métamorphose de gradient Une circulation d'air s'établit au sein de la couverture nivale, de nouveaux cristaux se développent alors, dits en gobelets en raison de leur forme en pyramide creuse qui forment une couche particulièrement instable.

La métamorphose de fonte survient quand la température superficielle de la neige atteint $0^{\circ}$ sous l'influence du soleil, de la pluie ou du réchauffement de l'air. L'eau liquide absente des précédentes métamorphoses fait son apparition, la neige devient humide et dense. Cette eau arrondit les grains anguleux et les cristaux grossissent par incorporation d'eau disponible dans les espaces intergranulaires. Le réchauffement diurne désolidarise le manteau neigeux, tandis qu'il $y$ a regel durant la nuit.

La connaissance de l'état du manteau nival est fondamentale dans la prévision des avalanches.

\section{La prévision des avalanches}

\section{Le rôle de Météo-France}

Dans les départements montagnards, Météo-France diffuse un bulletin "montagne et neige" qui fournit des données sur le temps du jour et l'enneigement des massifs.

Néanmoins le bulletin de risque d'avalanches demeure le plus précis et le plus utile. Son élaboration s'appuie sur le réseau nivométéorologique.

C'est un réseau saisonnier dont la période d'activité est calquée sur l'ouverture des stations de sports d'hiver. Que mesure-t-on dans un réseau nivométéorologique? 
- En surface: les données météorologiques

- la nébulosité et les types de nuages

- la force et la direction du vent

- le temps actuel

- la température de l'air (actuelle, maxi, mini)

- la lame d'eau tombée en 24 h. (ou équivalent d'eau en neige)

Depuis 1982 des stations automatiques de mesures à haute altitude existent, leurs données sont transmises par une balise à un satellite météorologique. Elles fournissent.

- Les paramètres nivologiques à savoir:

- l'épaisseur de neige au sol

- l'épaisseur de neige fraîche tombée en $24 \mathrm{~h}$

- la température de la neige à $10 \mathrm{~cm}$ de la surface

- l'état de surface de la neige

- la mesure de la résistance de la couche superficielle

- l'observation du transport de la neige par le vent

- l'activité avalancheuse observée

- Dans certains cas sont aussi mesurés

- l'humidité de l'air

- la teneur en eau liquide de la neige

- le type et le diamètre des grains de surface

- la masse volumique de la couche de neige

- Les mesures du manteau nival en profondeur:

Une fois par semaine des observateurs réalisent des sondages dans la couverture nivale qui permettent de connaître l'état du manteau nival sur toute son épaisseur. Un profil stratigraphique peut être réalisé à partir de l'analyse de

- la résistance à l'enfoncement (sondage par battage)

- le profil vertical de température dans le manteau nival

- l'identification des strates: grains, dureté, humidité, masse volumique

- la teneur en eau liquide, résistance au cisaillement au moins dans certains cas.

\section{Des bulletins d'estimation du risque d'avalanche (BRA)}

La gestion du risque d'avalanche repose sur un partenariat entre les stations de sport d'hiver et météo-France. Entre 1970 et 1987 la prévision a été assurée par le Centre d'études de la neige ou CEN sur l'ensemble des massifs montagneux français. Depuis 1987 les centres météorologiques alpins, pyrénéens et corses, assurent ce travail. L'information "neige et avalanche" est fournie parles bulletins départementaux. Chaque département a été découpé en zones, il en existe vingt trois dans les Alpes, onze dans les
Pyrénées et deux en Corse. C'est à l'échelle de ces secteurs que I'on nomme aussi "massifs" et dont la surface atteint entre 500 et $1000 \mathrm{~km}^{2}$, que sont indiquées les informations sur les conditions de neige et de risque d'avalanche Les Bulletins d'estimation des Risques d'avalanches (BRA) qui sont diffusés quotidiennement en hiver, fournissent des renseignements sur l'état du manteau nival et sur le risque d'avalanche envisagé en dehors des pistes balisées et ouvertes, en référence à l'échelle européenne des risques d'avalanches qui compte cinq niveaux ( 1 faible, 2 modéré, 3 marqué, 4 fort, 5 très fort tableau $n^{\circ} 3$ ).

Concernant l'échelle du risque, Météo-France rappelle que "l'indice chiffré ne fournit qu'une information limitée". Le but de Météo-France n'est pas d'interdire, nid'autoriser la pratique de la montagne. Le bulletin est un outil d'aide à la décision permettant à chacun d'adapter son comportement aux conditions de neige et aux risques prévus.

\section{La modélisation}

Elle apparaît aussi comme un outil d'aide à la décision pour le prévisionniste.

Le modèle SAFRAN fournit des données météorologiques massif par massif, il utilise les informations des postes nivométéorologiques, des stations automatiques. C'est un modèle de "spatialisation" des variables météorologiques: les paramètres météo nécessaires à CROCUS sont extrapolés par SAFRAN. CROCUS permet de visualiser l'état du manteau nival d'un massif un jour donné, par tranches d'altitudes de 300 $\mathrm{m}$, en différentes expositions et pour différentes pentes, $0^{\circ}, 20^{\circ}$ et $40^{\circ}$. Cela permet de dépasser la contrainte liée à la fréquence seulement hebdomadaire des sondages de battage réalisés sur le terrain par des observateurs du réseau nivométéorologique.

Les modèles permettent d'a voir des informations répétées et précises sur la surface de la neige et sur l'état de la couche, tassement, densité, humidification.

MEPRA est un système expert d'estimation du risque d'avalanche. Il calcule d'autres paramètres comme la résistance au cisaillement, en précisant les types de grains. MEPRA analyse la stabilité mécanique de chaque couche. Ce modèle effectue un diagnostic à l'échelle d'un massif montagneux sur l'ampleur des risques d'avalanche en fonction de l'altitude et de l'exposition.

Ces modèles certes fort utiles, montrent pourtant certaines limites, ils ne prennent pas en compte le vent comme agent mécanique, et pas davantage les données géographiques expliquant qu'un massif ne reçoit pas sur toute sa surface, des tranches de précipi- 
Tableau $n^{\circ} 3$

Echelle européenne des risques d'avalanches.

\begin{tabular}{|c|c|c|}
\hline Indice du risque & Stabilité du manteau neigeux & Probabilité de déclenchement \\
\hline $\begin{array}{c}\text { Faible } \\
1\end{array}$ & $\begin{array}{l}\text { Le manteau neigeux est bien stabilisé dans la } \\
\text { plupart des pentes. }\end{array}$ & $\begin{array}{l}\text { Les déclenchements d'avalanches ne sont possibles } \\
\text { que dans de très rares pentes raides }(*) \text {, surtout par } \\
\text { forte surcharge }(* * *) \text {. Seules des coulées ou de petites } \\
\text { avalanches peuvent se produire spontanément. }\end{array}$ \\
\hline $\begin{array}{l}\text { Limité } \\
2\end{array}$ & $\begin{array}{l}\text { Dans quelques }(* *) \text { pentes suffisamment raides, } \\
\text { le manteau neigeux n'est que modérément stabilisé. } \\
\text { Ailleurs, il est bien stabilisé. }\end{array}$ & $\begin{array}{l}\text { Déclenchements d'avalanches possibles surtout par } \\
\text { forte surcharge }(* * *) \text { et dans quelques pentes dont les } \\
\text { caractéristiques sont généralement décrites dans le } \\
\text { bulletin. Des départs spontanés d'avalanches de grande } \\
\text { ampleur ne sont pas à attendre. }\end{array}$ \\
\hline $\begin{array}{l}\text { Marqué } \\
3\end{array}$ & $\begin{array}{l}\text { Dans de nombreuses }(* *) \text { pentes suffisamment } \\
\text { raides, le manteau neigeux n'est que modérément } \\
\text { à faiblement stabilisé. }\end{array}$ & $\begin{array}{l}\text { Déclenchements d'avalanches possibles parfois même } \\
\text { par faible surcharge et dans de nombreuses pentes } \\
\text { dont les caractéristiques sont généralement décrites } \\
\text { dans le bulletin. Dans certaines situations, quelques } \\
\text { départs spontanés d'avalanches de taille moyenne, et } \\
\text { parfois assez grosses sont possibles. }\end{array}$ \\
\hline $\begin{array}{c}\text { Fort } \\
4\end{array}$ & $\begin{array}{l}\text { Le manteau neigeux est faiblement stabilisé } \\
\text { dans la plupart }\left(^{* *}\right) \text { des pentes suffisamment } \\
\text { raides. }\end{array}$ & $\begin{array}{l}\text { Déclenchement d'avalanches possibles même par } \\
\text { faible surcharge (****) dans la plupart des pentes } \\
\text { suffisamment raides. Dans certaines situations, de } \\
\text { nombreux départs spontanés d'avalanches de taille } \\
\text { moyenne, et parfois grosses, sont à attendre. }\end{array}$ \\
\hline \multirow[t]{2}{*}{$\begin{array}{l}\text { Très fort } \\
5\end{array}$} & L'instabilité du manteau neigeux est généralisée. & $\begin{array}{l}\text { De nombreuses et grosses avalanches se produisant } \\
\text { spontanément sont à attendre y compris en terrain } \\
\text { peu raide. }\end{array}$ \\
\hline & $\begin{array}{l}\text { (*) Pentes particulièrement propices aux avalan- } \\
\text { ches, en raison de leur déclivité, la configuration } \\
\text { du terrain, la proximité de la crête... } \\
\text { (**) Les caractéristiques de ces pentes sont } \\
\text { généralement précisẹes dans le bulletin: altitude, } \\
\text { exposition, topograph. } \\
\text { Le terme "déclenchement" concerne les avalanches } \\
\text { provoquées par surcharge, notamment par le ou } \\
\text { les skieurs. }\end{array}$ & $\begin{array}{l}\text { (***) Surcharge indicative: } \\
\text { - forte: par exemple, skieurs groupés; } \\
\text { - faible: par exemple, skieur isolé, piéton } \\
\text { Le terme "départ spontané" concerne les avalanches } \\
\text { qui se produisent sans action extérieure. }\end{array}$ \\
\hline
\end{tabular}

tations homogènes, les chutes de neige peuvent en effet varier du simple au triple à quelques $\mathrm{km}$ de distance.

Ces modèles fonctionnent assez bien dans les Alpes malgré les limites évoquées, un peu plus difficilement pour les Pyrénées où le découpage climatique est plus difficile en raison de l'aspect de la chaîne, et où les données météo sont à la fois françaises, espagnoles et andorranes.

\section{Les travaux de protection, le génie paravalanche}

La défense porte sur deux aspects: la protection en cas de départ de la neige et la fixation de la neige pour réduire sa mise en mouvement.
La protection contre l'écoulement avalancheux consiste à tenter de détourner l'avalanche, de freiner ou d' arrêter la neige, de renforcer les constructions, de mettre en place différents équipements permettant par exemple à l'avalanche de passer au-dessus d'une route: galeries, tunnels, "tourne", digues de déviation, ou levée en maçonnerie détournant la coulée de neige, il peut s'agir d' une étrave, forme de $\mathrm{V}$ qui peut être placée en amont d'un bâtiment ou d'une installation à protéger.

Des ouvrages de freinage et d'arrêt existent aussi: des tas freineurs, buttes en terre ou en maçonnerie disposées en réseau freinent l'avalanche. Mais si une première avalanche survient suivie d'une seconde celle-ci peut passer au-dessus du secteur traité et comblé. Des dents freineuses sont des ouvrages 
pyramidaux en béton armé, hauts de plusieurs mètres qui offrent les mêmes caractéristiques que le tas freineur, ces dents sont plus grandes que les tas freineurs, ce qui leur permet de résister à de fortes pressions.

Des détecteurs routiers d'avalanches annoncent l'arrivée d'une avalanche sur une voie de circulation. Un contact électrique est déclenché sur le versant par le passage d'une avalanche au-dessus de la route à protéger. L'élément déclencheur est placé $2 \mathrm{~m}$ audessus du sol de manière que l'avalanche l'affecte mais que le passage d'animaux ne le déclenche pas. Ce dispositif est valable pour les écoulements de neige dense bien canalisés, mais il faut vérifier le bon fonctionnement du système.

\section{La fixation de la neige dans les lieux de départ potentiel}

\section{+ Le couvert végétal}

Il s'agit de fixer le manteau nival, sur des pentes raides et des lieux d'accès difficile. Cela peut être effectué grâce à l'aménagement de banquettes étroites (1 m de largeur) destinées à freiner la reptation de la neige et son glissement au sol. Leur effet est d'autant plus grand que ces banquettes sont boisées, ce qui ne peut être le cas au-dessus d'une certaine altitude correspondant aux limites de la forêt et de l'arbre.

De manière générale la revégétalisation est fondamentale. La politique de Restauration des Terrains de Montagnes conduite depuis 1882 dans les massifs français, permet de souligner la validité de ces pratiques. Dans le haut Conflent (Pyrénées Orientales) certains versants ont été dans le passé très affectés par des phénomènes avalancheux qui ont cessé depuis le début du XX ème siècle, probablement parce que le coefficient de rugosité a augmenté dans les couloirs, en raison de l'extension de la forêt. Ainsi équipés ces couloirs ne sont plus les beaux toboggans qu'ils étaient à la fin du XVIII ème siècle (G. Soutadé).

Néanmoins certaines avalanches partent de secteurs installés dans le domaine supra-forestier et ravagent les espaces forestiers situés à plus basse altitude. Une utilisation insuffisante des prairies d'altitude (diminution du pâturage et développement d'herbes longues formant plan de glissement) favorise le glissement du couvert nival.

\section{+ Les types d' aménagements pourfixerle manteau neigeux}

Des râteliers, ouvrages en métal (aluminium) ou en bois composés de traverses verticales non jointives sont placés en ligne continue, parallèlement aux courbes de niveau pour fixer la neige. Des filets, souples avec des mailles d'une vingtaine de centimètres, des barrières à neige métalliques ou en bois de 4 à 5 $\mathrm{m}$ de hauteur, limitent localement la formation de congères. Elles sont placées face au vent. Il existe aussi des barrières mobiles, qui s'orientent face au vent dominant. Les Vire-vents sont des facettes en forme de trapèze en bois, hautes de plusieurs mètres, qui perturbent l'écoulement de l'air et créés des tourbillons qui érodent localement le manteau nival empêchant l'élaboration de congères.

\section{Les aspects techniques de la protection: le déclen- chement}

Le déclenchement artificiel des avalanches, peut être effectué dans le cadre du Plan d'Intervention pour le Déclenchement des Avalanches (PIDA) document proposé par le Maire visé par le Préfet qui précise les secteurs concernés par des tirs préventifs, la procédure utilisée, les itinéraires à suivre, les zones interdites, les intervenants, les consignes de tir.

Le déclenchement peut être manuel:1 'explosion a lieu sur le manteau neigeux, son efficacité est faible, comme son coût. Cependant, la méthode est difficilement applicable quand l'accès du terrain est difficile.

Des lanceurs pneumatiques de flèches explosives sont aujourd'hui utilisées. Il s'agit d'un tube de $1,8 \mathrm{~m}$ de long contenant un mélange liquide explosif de $2 \mathrm{~kg}$ qui explose juste avant d'atteindre le sol. Ce type de canon fonctionne à l'azote comprimée, il projette une flèche creuse contenant un explosif liquide actif pendant quelques heures seulement. Sa portée est d'environ $1800 \mathrm{~m}$ et $350 \mathrm{~m}$ de dénivelée.

La méthode duCATEX utilise un câble transporteur d'explosif, soutenu par des pylônes, une sorte de téléski qui peut monter 2 à $10 \mathrm{~kg} \mathrm{~d}$ 'explosif, et permet d'en placer quelques grammes juste au-dessus du manteau neigeux, dans plusieurs couloirs proches. Il est employéen France depuis vingt ans. Les montagnes françaises disposent de 160 installations utilisées pour protéger les pistes et les routes. Il est concurrencé par le GAZEX: une installation faite d'un tube où est enflammé un mélange détonant de propane et d'oxygène. Il faut installer une réserve de gaz avant l'hiver, dans un abri, ainsi qu' une commande électrique. Il existe désormais une centaine de tubes explosifs de ce type en France. C'est un dispositif dont l'emploi est peu coûteux, très souple et discret.

\section{La prévention: Ia dimension spatiale. Carto- graphie des avalanches et PPR}

La prévention impose une connaissance des avalanches passées, de leur extension, de leur fréquence et de l'intensité du phénomène. 


\section{Les avalanches historiques}

L'étude des avalanches historiques apparaît fondamentale. Ces indices d'avalanches anciennes se situent soit sur le terrain (cônes d'avalanches), soit dans les archives ou la mémoire des populations.

Ces informations doivent conduire à établir les localisations des avalanches passées (trajectoires), leur ampleur, leur fréquence. Il est important de tenter d'établir l'avalanche majeure de référence pour envisager le zonage.

Dès 1900 l'inspecteur général des eaux et Forêts, Mougin, demandait aux préposés du service des eaux et Forêts de Savoie d'établir un relevé des couloirs d'avalanches et de consigner d'hiver en hiver toutes les avalanches se produisant dans le massif savoyard. En 1920 cette mesure fut élargie aux Hautes Alpes et à l'Isère puis à l'ensemble du massif alpin et des Pyrénées. Ainsi a été mise en place l'Enquête permanente sur les avalanches (EPA), prolongée ensuite dans le cadre de la division nivologique du CEMAGREF.

L'administration des Eaux et forêts a donc tenu à jour des "cahiers d'avalanches", les rapports des agents des eaux et forêts étant résumés et consignés dans des documents qui sont restés du même type de 1900 à 1971.

Ces carnets fournissent des informations sur les matériaux mobilisés par les avalanches, en 1914, 3383 mètres cubes de bois ont été déplacés dans la vallée de Chamonix. Les jeunes pousses d'arbres ont été détruites, les forêts d'épicéas et de mélèzes affectées. Les dégâts aux habitations et aux infrastructures, chalets, lignes électriques, voies ferrées, ont été soigneusement consignés.

L'agent qui effectue l'observation doit reporter ses observations sur un fond de carte topographique au $1 / 25000$ et notamment les contours précis des couloirs d'avalanches. Les informations de l'EPA sont reportées sur deux fichiers désormais informatisés, RAVA et SITE, le premier s'intéresse à la description de l'avalanche, le second aux données plus géographiques (altitude, coordonnées Lambert, trajet).

A la suite de la catastrophe de Val d'Isère en 1970 la Commission interministérielle sur la sécurité des stations de Montagne a recommandé la réalisation d'une carte, inventaire des avalanches indispensables dans les services publics.

\section{Les Cartes de Localisation Probable des Avalan- ches CLPA}

Le Ministère de l'Agriculture s'est vu confier la réalisation de ce document en 1970. Aujourd'hui plus de 600000 ha ont été cartographiés dans les Alpes et les Pyrénées.

Il s'agit de cartes inventaires des avalanches qui se sont déjà produites, ces documents qui concernent plus les aléas que des risques s.s. ne prennent pas en compte la fréquence et l'intensité des phénomènes. Ils qui n'ont pas de valeur réglementaire.

La réalisation de ces documents repose sur une étude par photo-interprétation (photo aériennes au 1/30000) des traces géomorphologiques des avalanches: il s'agit de localiser les couloirs, d'envisager les marques dans la végétation, arbres cassés, ruines, pierres.

L'enquête de terrain et les documents d'archives constituent une autre étape de la réalisation.

Ces cartes constituent un état des lieux, dressé au 25000 .

La présentation cartographique associe des zones de couleur à plat qui composent les secteurs où les avalanches sont les mieux définies, des flèches quand le phénomène est étroit pour être représenté en à plat, des zones en hachures correspondant à des secteurs pouvant être affectés par des avalanches qui ne sont pas distinguées de leur voisine par une limite précise. Des tiretés correspondent aux lieux où les informations obtenues sont vagues, contradictoires.

Il n'y a pas de hiérarchie entre ces différentes représentations. les hachures ou les flèches n'indiquent pas un secteur moins dangereux que les à plats.

Les informations provenant de la photo-interprétation sont portées en orange, celles issues de l'enquête en magenta. Les travaux de protection figurent en surcharge noire.

Les CLPA sont des instruments que les Maires ou des services administratifs ou techniques doivent consulter, mais elles ne sont pas opposables au tiers.

\section{Les Plans des Zones Exposées aux Avalanches PZEA, les Plans de Préventions des Risques PPR}

Les PZEA ont été lancés par la commission interministérielle d'étude sur la sécurité des stations de montagne qui a recommandé dans son rapport au conseil des ministres en 1970 l'établissement des PZEA, destinés à cerner les secteurs dangereux d'où les constructions devaient être exclues. Ce document établi à grande échelle 1/1000 à 1/5000 n'a aucune valeur juridique.

En 1982 ont été instaurés les Plans d'exposition aux risques (PER) qui marquaient la volonté de l'Etat de renforcer la prévention. L'Etat devant élaborer et mettre en application ces plans qui déterminent pour chaque commune concernée, les zones ex posées et les techniques de prévention à mettre en oeuvre. 
Ces PER constituaient une servitude d'utilité publique et s'imposaient aux plans d'occupation des sols auxquels ils devaient être annexés. Le PER permettait de délimiter les zones concernées par les risques à partirdes cartes de localisation probable des avalanches, de définir ou de prescrire des mesures de prévention. Trop contraignants, trop longs et trop coûteux à réaliser, les PER ont été remplacés par les PPR.

La loi du 2 février 1995 relative au renforcement de la protection de l'environnement ou loi Barnier a confié à l'Etat la réalisation de Plans de Prévention des Risques, dont l'objet est de délimiter les zones exposées, les secteurs où une activité aurait pour effet d'accroître les risques. Ils doivent définir les mesures à adopter dans les zones à risques par les propriétaires, exploitants et utilisateurs (précautions architecturales, limitation de la densité d'occupation, de la hauteur des immeubles). La réalisation du document impose d'effectuer une carte d'aléas, qui présente les processus selon leur intensité, faible, moyen, fort sur un fond au $1 / 10000$ ou $1 / 25000$. L'étude des enjeux, ce que l'on nomme parfois la vulnérabilité, conduit à une évaluation des populations en dangers, au recensement desétablissements recevant du public (hôpitaux, écoles...), à l' identification des axes de circulation.

Tableau n ${ }^{\circ} 4$

Les acteurs institutionnels de la gestion des risques

\section{ECHELLE LOCALE (Commune)}

\section{LE MAIRE}

Représentant de l'Etat dans sa commune depuis 1884

Les lois de décentralisation 1982-1983 augmentent sa responsabilité car il deûve les permis de construire.

\section{Pouvoir de police}

\section{Prévention}

Signalisation de l'affichage

Information des populations

Informations sur les mesures de sauvegarde qui ont été prises pour lutter contre les risques

Prescription des travaux de prévention

En cas de danger le maire doit alerter le préfet.

\section{Pouvoir d'Urbanisme}

Elaboration des POS qui depuis 1987 doivent prendre en compte les risques

Refus possible du permis de construire

\section{Compétence au titre de législations spécifiques}

Le maire a obligation pour certains bâtiments et dans le cadre de sa compétence à délivrer un permis de construire de consulter les organismes de sécurité civile ou une commission de sécurité pour s'assurer que les travaux réalisés sont conformes aux normes de sécurité.

\section{ECHELLE DE L'ETAT ET DES SERVICES DÉCONCENTRÉS \\ LE PREFET}

\section{Pouvoir de police}

Pouvoir de substitution, en cas de carence du maire quand plusieurs communes sont concernées par un risque

\section{Pouvoir administratif}

Contrôle de la légalité des actes administratifs émanant de la commune, avec le concours des services concernés de l'Etat.

"Le porter à connaissance": lors de l'élaboration des documents d'urbanismes (par le maire), le préfet doit porter à connaissance des communes toutes les informations dont il dispose en matière de risques. il a pour mission de collecter toutes les informations sur ce sujet

Le projet prend l'arrêt de prescription et conduit la réalisation d'un PPR peut mettre en oeuvre la procédure d'expropriation dans le cadre du PPR 
Le plan de zonage, le PPR proprement dit, délimite les secteurs d'interdiction, les prescriptions réglementaires. Si le propriétaire d'un bien privé n'a pas appliqué le règlement du $\mathrm{PPR}$, notamment la mise en conformité du bâti, l'assurance ne couvrira pas les frais pouvant résulter d'une catastrophe.

Le périmètre mis à l'étude après prescription du préfet vise en principe un bassin de risque (et non seulement la commune). L'arrêté peutêtre intercommunal si nécessaire.

La loi permet de rendre opposable par anticipation des dispositions qui n'ont pas encore fait l'objet d'un débat public, en cela le PPR diffère du PER. Cela peut conduire à arrêter des projets d'urbanisation, de constructions si nécessaire.

Le projet de PPR est soumis à un ensemble de consultations: conseils municipaux dans les communes concernées, organismes divers selon la nature des espaces et des aménagements: chambre d'agriculture, centre régional de la propriété forestière.

Sur l'ensemble des montagnes françaises, 158 PPR "avalanches" avaient été approuvés, 60 environ étaient en cours d'élaboration à la fin de 1999.

\section{Bibliographie}

Les apports de la recherche scientifique à la sécurité neige.

Glace et avalanche. Actes du colloque Chamonix 30 mai-3 juin 1995.

KERT, Ch. (1999) - Rapport sur les techniques de prévision et de prévention des risques naturels en France. Assemblée Nationale et Sénat, rapport no 1540 et n ${ }^{\circ} 312.419$ p.

Revue Neige et Avalanches.

Divers articles de la Revue de géographie alpine. 
